\title{
Some New Characterizations of Markov-Bernoulli Geometric Distribution Related to Random Sums
}

\author{
M. Gharib ${ }^{1}$, M. M. Ramadan ${ }^{2,3}$ \& Kh. A. H. Al-Ajmi ${ }^{1}$ \\ ${ }^{1}$ Department of Mathematics, Faculty of Science, Ain Shams University, Abbasia, Cairo, Egypt \\ ${ }^{2}$ Department of Marketing Administration, Community College, Taif University, Taif, Saudi Arabia \\ ${ }^{3}$ Department of Mathematics, Faculty of Education, Ain Shams University, Roxy, Cairo, Egypt \\ Correspondence: M. Gharib, Department of Mathematics, Faculty of Science, Ain Shams University, Abbasia, \\ Cairo, Egypt. E-mail: mgmahmoud51@gmail.com
}

Received: June 22, 2014 Accepted: July 13, 2014 Online Published: July 28, 2014

doi:10.5539/ijsp.v3n3p138～URL: http://dx.doi.org/10.5539/ijsp.v3n3p138

\begin{abstract}
The Markov-Bernoulli geometric distribution is obtained when a generalization, as a Markov process, of the independent Bernoulli sequence of random variables, is introduced. In this paper, new characterizations of the Markov-Bernoulli geometric distribution, as the distribution of the summation index of randomly truncated nonnegative integer valued random variables, are given in terms of moment relations of the sum and summands. The achieved results generalize the corresponding characterizations concerning the usual geometric distribution.
\end{abstract}

Keywords: Markov-Bernoulli geometric distribution, random sum, characterization, random truncation, moments, Euler differential equation

\section{Introduction}

Many basic counting distributions are defined on a se-quence of independent identically distributed (iid) Bernoulli random variables (rv's). Many other distributions are defined by compounding and mixing. Another way of obtaining new discrete distributions is to define the counting distributions related to some Markov chain. Assuming some dependency in the sequence of Bernoulli rv's gives an additional parameter by which the Bernoulli model could be a more realistic model in practice. Edwards (1960) proposed a generalization of the sequence of independent Bernoulli trials by considering the success probability evolves over time according to a Markov chain. In other words, let $X_{1}, X_{2}, \ldots$ be a sequence of Bernoulli rv's with the following one step transition probabilities matrix

$$
\begin{aligned}
& X_{i+1} \\
& 0 \quad 1 \\
& \begin{array}{ll}
X_{i} & 0 \\
& 1
\end{array}\left[\begin{array}{cc}
1-(1-\rho) p & (1-\rho) p \\
(1-\rho)(1-p) & \rho+(1-\rho) p
\end{array}\right]
\end{aligned}
$$

and initial distribution:

$$
P\left(X_{1}=1\right)=p=1-P\left(X_{1}=0\right),
$$

where $p \in[0,1]$ and $\rho \in[0,1]$. The additional parameter $\rho$ is the correlation coefficient between $X_{i}$ and $X_{i+1}$, and is, also, called the persistent indicator of the initial state of the system (1.1) (Wang, 1981).

The sequence $X_{1}, X_{2}, \ldots$ with the transition matrix (1.1) and the given initial distribution is called the MarkovBernoulli model(MBM) or the Markov modulated Bernoulli process (Özekici, 1997). Numerous researchers have studied the MBM from the various aspects of probability, statistics and their applications, in particular the classical problems related to the usual Bernoulli model (Anis \& Gharib, 1982; Arvidsson \& Francke, 2007; Čekanavičius \& Vellaisamy, 2010; Gharib \& Yehia, 1987; Inal, 1987; Maillart et al., 2008; Minkova \& Omey, 2011; Omey et al., 2008; Özekici, 1997; Özekici \& Soyer, 2003; Pacheco et al., 2009; Pedler, 1980; Pires \& Diniz, 2012; Satheesh et al., 2002; Xekalaki \& Panaretos, 2004 and others). Further, due to the fact that the MBM operates in a random environment depicted by a Markov chain so that the probability of success at each trial depends on the state of the environment, this model has a wide variety of applications include, but not limited, reliability modeling 
(where system and components function in a randomly changing environment), non-life insurance, matching DNAsequences, disease clustering, traffic modeling, the occupation and waiting times problems in two state Markov chains, reconstructing patterns from sample data and statistical ecology (Arvidsson \& Francke, 2007; Chang \& Zeiterman, 2002; John, 1971; Özekici, 1997; Özekici \& Soyer, 2003; Pacheco et al., 2009; Pedler, 1980; Pires \& Diniz, 2012; Switzer, 1967, 1971; Wang, 1981; Xekalaki \& Panaretos, 2004).

The Markov-Bernoulli geometric (MBG) distribution has been obtained by Anis and Gharib (1982) in an earlier detailed study of MBM. If $E_{i} ; i=0 ; 1$ are the states of the system (1.1) and if $N$ is a rv representing the number of trials necessary for the system to be in state $E_{1}$ for the first time, then the probability mass function (pmf) of the rv $N$ is given by:

$$
P(N=n)= \begin{cases}p, & n=1 \\ (1-p) a^{-1}\left(1-a^{-1}\right)^{n-2}, & n \geq 2,\end{cases}
$$

where, $a=1 /[(1-\rho) p]=E(N)+\rho /(1-\rho)$.

The distribution (1.2) is the MBG distribution. It represents a generalization of the usual geometric distribution ( $\rho=$ 0 ). In the past works on the MBM some characterizations for the MBG distribution (1.2) are achieved (Minkova \& Omey, 2011; Yehia \& Gharib, 1993). The random sum of independent identically distributed (iid) nonnegative rv's; where the summation index is a geometric rv; is called a geometric compounding of the underlying rv's. Such a compounding mechanism is closely related to the rarefaction (or the thinning version) of a renewal process and has practical applications to the traffic theory, reliability and ecology problems involving rare events (Gnedenko \& Korolev, 1996). These applications motivated many researchers to characterize such random sums, see e.g. Gharib et al. (2003), Khalil et al. (1991), Milne and Yeo (1989) and the references therein.

Let $\left\{X_{n} ; n \geq 1\right\}$ be a sequence of iid integer-valued rv's with pmf: $p_{k}=P\left(X_{1}=k\right)<1, k=0,1,2, \ldots$; and let $\left\{Y_{n} ; n \geq 1\right\}$ be another sequence of iid integer-valued rv's with pmf: $q_{k}=P\left(Y_{1}=k\right)<1, k=0,1,2, \ldots, \sum_{k=0}^{\infty} q_{k}=1$. The two sequences $\left\{X_{n}\right\}$ and $\left\{Y_{n}\right\}$ are assumed independent. Define the integer-valued $\operatorname{rv} N=\inf \left\{n \geq 1: X_{n}<Y_{n}\right\}$. Clearly $N-1$ has a geometric distribution with pmf: $P(N-1=k)=p^{k}(1-p), k=0,1,2, \ldots$, where $p=P\left(X_{1} \geq Y_{1}\right)$ and $p \in(0,1)$.

In this paper, we are interested in characterizing the random sum:

$$
Z=\sum_{k=0}^{N-1} Y_{k}+X_{N}, \quad Y_{0}=0
$$

introduced by Khalil et al. (1991).

The rv $Z$ represents the truncated sum until the moment where for the first time the process $\left\{Y_{n} ; n \geq 1\right\}$ has greater jump than the corresponding jump of the process $\left\{X_{n} ; n \geq 1\right\}$. The sequence $\left\{Y_{n}\right\}$ is called the truncating process (Gharib et al., 2014). In queuing systems with unreliable server, $Z$ can be interpreted as the total time duration of the unreliable server until the successful finish of the service if the corresponding duration without breakdowns is previously known (Dimitrov et al., 1991). The random sum (1.3) also arises in some models such as unreliable computing systems, multiclients computer service, data transmissions, constructing data and so on, where in a random environment some undesirable events (breakdowns, interruptions, etc.) arise and lead to process interruption or to incorrect final results (Duda, 1983; Koren et al., 1986).

Throughout the paper, we write $P_{U}(s)=E\left(s^{U}\right) ;|s| \leq 1$, to denote the probability generating function (pgf) of an integer-valued rv $U, W \sim M B G(\alpha, c)$ to denote a MBG distributed rv $W$ with pmf:

$$
P(W=k)= \begin{cases}1-\alpha, & k=0 \\ \alpha c^{-1}\left(1-c^{-1}\right)^{k-1}, & k \geq 1,\end{cases}
$$

where, $c=1 /[(1-\rho)(1-\alpha)], \alpha \in(0,1), \rho \in[0,1]$, and $U \stackrel{d}{=} V$ to denote a $\mathrm{rv} U$ having the same distribution as a rv $V$. Let $I(A)$ be the indicator of a set $A$ and put

$$
\begin{aligned}
& P_{1}(s)=E\left[s^{X_{1}} I\left(X_{1}<Y_{1}\right)\right]=\sum_{\mathrm{k}=0}^{\infty} p_{k} s^{k} \sum_{\gamma=k+1}^{\infty} q_{\gamma}, \\
& Q_{1}(s)=E\left[s^{Y_{1}} I\left(X_{1} \geq Y_{1}\right)\right]=\sum_{\mathrm{k}=0}^{\infty} q_{k} s^{k} \sum_{\gamma=k}^{\infty} p_{\gamma} .
\end{aligned}
$$


The pgf of $Z$ in (1.3) is given by:

$$
P_{Z}(s)=P_{1}(s) /\left[1-Q_{1}(s)\right]
$$

(cf. Khalil et al., 1991).

In the following, we list some results obtained by Gharib et al. (2014) which are of direct relevance to the development of the results of the present paper. Consider the random sum $Z$ defined by (1.3).

Lemma 1.1 Assume that $Y_{1} \sim M B G(\alpha, \rho)$, for $\alpha \in(0,1)$ and $\rho \in[0,1]$, Then the pgf of $Z$ is given by

$$
P_{Z}(s)=\frac{\left[(1-t s) P_{X_{1}}(t s)\right]}{\left[1-\left\{1-(1-t) P_{X_{1}}(t s)\right\} s\right]}, \quad|s| \leq 1
$$

and

$$
E(Z)=P_{Z}^{\prime}(1)=\frac{\left[1-P_{X_{1}}(t)\right]}{\left[(1-t) P_{X_{1}}(t)\right]},
$$

where $t=\rho+(1-\rho) \alpha$.

Lemma 1.2 Assume $X_{1} \sim M B G\left(\beta, \rho_{1}\right)$; for some parameters $\beta \in(0 ; 1)$ and $\rho_{1} \in[0,1]$, and $Y_{1}$ is arbitrarily non-negative integer-valued $r v$ with $q_{0}=P\left(Y_{1}=0\right)<1$, then $Z \stackrel{d}{=} X_{1}$.

Using the results of Lemma 1.1 and Lemma 1.2, Gharib et al. (2014) gave the following two characterizations of the MBG distribution.

Theorem 1.1 Assume $Y_{1} \sim M B G(\alpha, \rho)$, for some parameters $\alpha \in(0 ; 1)$ and $\rho \in[0,1]$, and $X_{1}$ is an arbitrarily non-negative integer-valued $r v$ with $p_{0}=P\left(X_{1}=0\right)<1$, Then $Z \stackrel{d}{=} X_{1}$ if and only if $X_{1} \sim M B G\left(\beta, \rho_{1}\right)$, for some $\beta \in(0 ; 1)$ and $\rho_{1} \in[0,1]$.

Theorem 1.2 Assume $Y_{1} \sim M B G(\alpha, \rho)$; for some $\alpha \in(0 ; 1)$ and $\rho \in[0,1]$ and $X_{1}$ is an arbitrarily non-negative integer-valued $r v$ with $p_{0}=P\left(X_{1}=0\right)<1$ : Then $X_{1} \sim M B G\left(\beta, \rho_{1}\right)$, for some $\beta \in(0 ; 1)$ and $\rho_{1} \in[0,1]$, if and only if $E(Z)=\beta /(1-D)$ where $D=\rho_{2}+\left(1-\rho_{2}\right) \beta, \rho_{2}=\rho_{1}$ t and $t=\rho+(1-\rho) \alpha$.

The organization of this paper is as follows. In section 2 , we derive certain differential equations associated with the random sum (1.3). Also, the unique solutions of these differential equations are given under certain initial conditions. In section 3, we provide three new characterizations of the MBG distribution related to the random sum (1.3) based on certain relations between the moments of $Z$ and/or $X_{1}$. Finally some concluding remarks are given.

\section{Preliminaries}

The following lemma derives the main differential equations that will be used in the proofs of the new characterizations of the MBG distribution.

Lemma 2.1 Let $\phi(t)=-1 / P_{X_{1}}(t), t=\rho+(1-\rho) \alpha, \rho \in[0,1], \alpha \in(0,1)$, and $\mu_{r}=E\left(Z^{r}\right), r=1,2,3,4$. Then

(i) $t \phi^{\prime}(t)+\mu_{1} \phi(t)=-\frac{1}{2}\left(\mu_{2}+\mu_{1}\right)(1-t)$.

(ii) $t^{2} \phi^{\prime \prime}(t)+2 \mu_{1} t \phi^{\prime}(t)+\left(\mu_{2}-\mu_{1}\right) \phi(t)=-\frac{1}{3}\left(\mu_{3}-\mu_{1}\right)(1-t)$.

(iii) $t^{3} \phi^{\prime \prime \prime}(t)+3 \mu_{1} t^{2} \phi^{\prime \prime}(t)+3\left(\mu_{2}-\mu_{1}\right) t \phi^{\prime}(t)+\left(\mu_{3}-3 \mu_{2}+2 \mu_{1}\right) \phi(t)=-\frac{1}{4}\left(\mu_{4}-2 \mu_{3}-\mu_{2}+2 \mu_{1}\right)(1-t)$.

Proof. First, rewrite (1.7) as:

$$
\left(1-s\left[1-(1-t) P_{X_{1}}(t s)\right]\right) P_{Z}(s)-(1-t s) P_{X_{1}}(t s)=0,
$$

where $t=\rho+(1-\rho) \alpha, \alpha \in(0,1), \rho \in[0,1]$ and $|s| \leq 1$.

(i) Differentiating (2.1) twice w.r.t. $s$, then setting $s=1$, and using the relations $P_{Z}(1)=1, P_{Z}^{\prime}(1)=\mu_{1}, P_{Z}^{\prime \prime}(1)=$ $\mu_{2}-\mu_{1}$, we obtain:

$$
2 t\left[1+(1-t) \mu_{1}\right] P_{X_{1}}^{\prime}(t)+(1-t)\left(\mu_{1}+\mu_{2}\right) P_{X_{1}}(t)-2 \mu_{1}=0 .
$$

Now, using (1.8), we have:

$$
\phi^{\prime}(t)=\frac{P_{X_{1}}^{\prime}(t)}{P_{X_{1}}^{2}(t)}=\left[1+(1-t) \mu_{1}\right] \frac{P_{X_{1}}^{\prime}(t)}{P_{X_{1}}(t)} .
$$


Hence, using the last relation, Equation (2.2) reduces to

$$
t \phi^{\prime}(t)+\mu_{1} \phi(t)=-\frac{1}{2}\left(\mu_{2}+\mu_{1}\right)(1-t) .
$$

Proving (i).

(ii) Differentiating (2.1) three times w.r.t. $s$, then setting $s=1$, and using the relations $P_{Z}(1)=1, P_{Z}^{\prime}(1)=\mu_{1} P_{Z}^{\prime \prime}(1)=$ $\mu_{2}-\mu_{1}, P_{Z}^{\prime \prime \prime}(1)=\mu_{3}-3 \mu_{2}+2 \mu_{1}$, we obtain:

$$
t^{2}\left[1+(1-t) \mu_{1}\right] P_{X_{1}}^{\prime \prime}(t)+t(1-t)\left(\mu_{2}+\mu_{1}\right) P_{X_{1}}^{\prime}(t)-\frac{1}{3}\left(\mu_{1}-\mu_{3}\right)(1-t) P_{X_{1}}(t)-\left(\mu_{2}-\mu_{1}\right)=0 .
$$

Now, using (1.8), we have:

$$
\phi^{\prime \prime}(t)=\frac{P_{X_{1}}^{\prime \prime}(t)}{P_{X_{1}}^{2}(t)}-2 \frac{\left[P_{X_{1}}^{\prime}(t)\right]^{2}}{P_{X_{1}}^{3}(t)}=\frac{\left[1+(1-t) \mu_{1}\right] P_{X_{1}}^{\prime \prime}(t)-2 \phi^{\prime}(t) P_{X_{1}}^{\prime}(t)}{P_{X_{1}}(t)} .
$$

From which we get:

$$
\left[1+(1-t) \mu_{1}\right] P_{X_{1}}{ }^{\prime \prime}(t)=\phi^{\prime \prime}(t) P_{X_{1}}(t)+2 \phi^{\prime}(t) P_{X_{1}}^{\prime}(t)
$$

Also, using (i), we have:

$$
\left(\mu_{2}+\mu_{1}\right)(1-t)=-2 t \phi^{\prime}(t)-2 \mu_{1} \phi(t)
$$

Now, using (2.4) and (2.5), Equation (2.3) reduces to

$$
t^{2} \phi^{\prime \prime}(t) P_{X_{1}}(t)-2 \mu_{1} t \phi(t) P_{X_{1}}^{\prime}(t)+\frac{1}{3}\left(\mu_{3}-\mu_{1}\right)(1-t) P_{X_{1}}(t)-\left(\mu_{2}-\mu_{1}\right)=0 .
$$

Finally, since $\phi(t) P_{X_{1}}^{\prime}(t)=-\phi^{\prime}(t) P_{X_{1}}(t)$ and $t=\rho+(1-\rho) \alpha$, then Equation (2.6) can be written as

$$
t^{2} \phi^{\prime \prime}(t)+2 \mu_{1} t \phi^{\prime}(t)+\left(\mu_{2}-\mu_{1}\right) \phi(t)=-\frac{1}{3}\left(\mu_{3}-\mu_{1}\right)(1-t) .
$$

Proving (ii).

(iii) Proof of this part is similar to that of (i) and (ii).

Remark 2.1 Lemma 2.1 reduces, for $\rho=0$, to Lemma 3 of Ghitany and Gharib (2005).

Remark 2.2 The differential equations derived in Lemma 2.1 has the following general form:

$$
a_{k} t^{k} \phi^{(k)}(t)+a_{k-1} t^{k-1} \phi^{(k-1)}(t)+\ldots+a_{1} t \phi^{(1)}(t)+a_{0} \phi(t)=g(t),
$$

where $a_{k}, a_{k-1}, \ldots, a_{0}$ are constants, $\phi^{(k)}(t)$ denotes the $k^{\text {th }}$ derivative of $\phi(t)$, and $g(t)$ is a non-zero function of $t$. This differential equation is known as the $k^{\text {th }}$ order nonhomogeneous Euler differential equation (Zwillinger, 1992, p. 235).

\section{Characterization Results}

The first characterization of the MBG distribution related to the random sum (1.3) is expressed in terms of the first two moments of $Z$.

Theorem 3.1 Assume $Y_{1} \sim M B G(\alpha, \rho)$, for some $\alpha \in(0 ; 1)$ and $\rho \in[0,1]$, and $X_{1}$ is arbitrary non-negative integer-valued $r v$ with $p_{0}=P\left(X_{1}=0\right)<1$. Then $X_{1} \sim M B G\left(\beta, \rho_{1}\right)$, for some $\beta \in(0 ; 1)$ and $\rho_{1} \in[0,1]$, if and only if

$$
\mu_{2}-\mu_{1}\left(2 \mu_{1}+1\right)=\frac{2 \beta \rho_{1} t}{(1-\beta)\left(1-\rho_{1} t\right)^{2}}
$$

where, $t=\rho+(1-\rho) \alpha$, and $E\left(Z^{i}\right)=\mu_{i}, i=1,2$ with $\mu_{2}<\infty$.

Proof. Necessity: Suppose $X_{1} \sim M B G\left(\beta, \rho_{1}\right)$, for some $\beta \in(0 ; 1)$ and $\rho_{1} \in[0,1]$. Then, by using Lemma 1.2, $Z \sim M B G\left(\beta, \rho_{2}\right)$, for some $\beta \in(0 ; 1)$ and $\rho_{2}=\rho_{1} t ; t=\rho+(1-\rho) \alpha$. Now, it is straight forward to see that condition (3.1) is satisfied upon substituting

$$
\mu_{1}=\frac{\beta}{1-D}, \quad \mu_{2}=\frac{\mu_{1}(1+D)}{1-D},
$$


where, $D=\rho_{2}+\left(1-\rho_{2}\right) \beta$.

Sufficiency: Suppose condition (3.1) is satisfied. This and (i) of Lemma 2.1 imply that

$$
t \phi^{\prime}(t)+\frac{\beta}{(1-\beta)\left(1-\rho_{1} t\right)} \phi(t)=-\frac{\beta}{(1-\beta)^{2}\left(1-\rho_{1} t\right)^{2}}(1-t), \quad t \in(0 ; 1), \text { and } \rho_{1} \in[0,1),
$$

subject to the initial condition $\phi(1)=-1$.

Now, the general solution of (3.2) is:

$$
\phi(t)=C_{1} t^{-\frac{\beta}{(1-\beta)}}\left(-1+\rho_{1} t\right)^{\frac{\beta}{(1-\beta)}}-\frac{(1-\ell t)}{(1-\beta)\left(1-\rho_{1} t\right)},
$$

where $C_{1}$ is an arbitrary constant and $\ell=\rho_{1}+\left(1-\rho_{1}\right) \beta$.

Now, using the initial condition $\phi(1)=-1$, we obtain $C_{1}=0$. Hence, the solution of (3.2) is given by:

$$
\phi(t)=-\frac{(1-\ell t)}{(1-\beta)\left(1-\rho_{1} t\right)}, \quad t \in(0 ; 1), \beta \in(0 ; 1), \text { and } \rho_{1} \in[0,1) .
$$

(For the uniqueness of this solution, see Zwillinger, 1992, p. 51).

Consequently, we have uniquely $\left(\phi(s)=-1 / P_{X_{1}}(s)\right)$.

$$
P_{X_{1}}(s)=\frac{(1-\beta)\left(1-\rho_{1} s\right)}{(1-\ell s)},|s| \leq 1, \beta \in(0 ; 1), \text { and } \rho_{1} \in[0,1) .
$$

Therefore, $X_{1} \sim M B G\left(\beta, \rho_{1}\right)$. This completes the proof of Theorem 3.1.

Remark 3.1 If $\rho_{1}=0$, Theorem 3.1 reduces to Theorem 3 of Ghitany and Gharib (2005) concerning the case of geometric distribution.

The second characterization of the MBG distribution related to the random sum (1.3) is given in terms of the first three moments of $Z$ and the first moment of $X_{1}$.

Theorem 3.2 Assume $Y_{1} \sim M B G(\alpha, \rho)$, for some $\alpha \in(0 ; 1)$ and $\rho \in[0,1]$, and $X_{1}$ is arbitrary non-negative integervalued $r v$ with $p_{0}=P\left(X_{1}=0\right)<1$ : Then $X_{1} \sim M B G\left(\beta, \rho_{1}\right)$, for some $\beta \in(0 ; 1)$, and $\rho_{1} \in[0,1]$, if and only if

$$
\left(2 \mu_{3}+\mu_{1}\right) \mu_{1}-3 \mu_{2}^{2}=0,
$$

and

$$
\mu_{2}-\mu_{1}-2 \mu_{1} v_{1}=\frac{2 \beta \rho_{1}(t-D)}{\left(1-\rho_{1}\right)(1-D)^{2}},
$$

where $D=\rho_{1} t+\left(1-\rho_{1} t\right) \beta, t=\rho+(1-\rho) \alpha, \alpha \in(0 ; 1), \rho \in[0,1], E\left(X_{1}\right)=v_{1}$, and $E\left(Z^{i}\right)=\mu_{i}, i=1,2,3$ with $\mu_{3}<\infty$.

Proof. Necessity: Suppose $X_{1} \sim M B G\left(\beta, \rho_{1}\right)$ for some $\beta \in(0 ; 1)$, and $\rho_{1} \in[0,1]$. Then according to Lemma 1.2, $Z \sim M B G\left(\beta, \rho_{2}\right)$, for some $\beta \in(0 ; 1)$ and $\rho_{2}=\rho_{1} t, t=\rho+(1-\rho) \alpha$. Now, it is straight forward to see that each of conditions (3.3) and (3.4) is satisfied upon substituting

$$
\mu_{1}=\frac{\beta}{1-D}, \quad \mu_{2}=\frac{\mu_{1}(1+D)}{1-D}, \quad \mu_{3}=\frac{\mu_{1}\left(D^{2}+4 D+1\right)}{(1-D)^{2}} \quad v_{1}=\frac{\beta}{1-\ell},
$$

where $D=\rho_{1} t+\left(1-\rho_{1} t\right) \beta, t=\rho+(1-\rho) \alpha$ and

$$
\ell=\rho_{1}+\left(1-\rho_{1}\right) \beta .
$$

Sufficiency: Suppose conditions (3.3) and (3.4) are satisfied. These and (ii) of Lemma 2.1, imply that

$$
t^{2} \phi^{\prime \prime}(t)+\frac{2 \beta t}{(1-D)} \phi^{\prime}(t)+\frac{2 \beta D}{(1-D)^{2}} \phi(t)=-\frac{2 \beta D}{(1-D)^{2}}(1-t), \quad t \in(0 ; 1), \beta \in(0 ; 1),
$$

subject to the initial conditions $\phi(1)=-1, \phi^{\prime}(1)=v_{1}=\frac{\beta}{1-\ell}$, where $\ell=\rho_{1}+\left(1-\rho_{1}\right) \beta$, and $\rho_{1} \in[0,1)$. 
Now, the general solution of Equation (3.5) is given by

$$
\phi(t)=t^{r_{1}}\left(-1+\rho_{1} t\right)^{r_{4}} C_{1}+\frac{(1-\beta)\left(-1+\rho_{1} t\right)^{r_{3}} t^{r_{2}}}{\sqrt{\beta^{2}-6 \beta+1}} C_{2}-\frac{(1-\ell t)}{(1-\beta)\left(1-\rho_{1} t\right)}
$$

where, $C_{1}$ and $C_{2}$ are arbitrary constants, $t=\rho+(1-\rho) \alpha, \ell=\rho_{1}+\left(1-\rho_{1}\right) \beta, \rho_{1} \in[0,1), r_{1,2}=\frac{1-3 \beta \pm \sqrt{\beta^{2}-6 \beta+1}}{2(1-\beta)}$ and $r_{3,4}=\frac{1+\beta \pm \sqrt{\beta^{2}-6 \beta+1}}{2(1-\beta)}$.

Now, using the initial conditions of Equation (3.5), we get the following two algebraic equations in $C_{1}$ and $C_{2}$

$$
\left(-1+\rho_{1}\right)^{r_{4}} C_{1}+\frac{(1-\beta)\left(-1+\rho_{1}\right)^{r_{3}}}{\sqrt{\beta^{2}-6 \beta+1}} C_{2}=0,
$$

and

$$
\left(-1+\rho_{1}\right)^{r_{4}-1}\left(\rho_{1}-r_{1}\right) C_{1}+\frac{(1-\beta)\left(-1+\rho_{1}\right)^{r_{3}-1}\left(\rho_{1}-r_{2}\right)}{\sqrt{\beta^{2}-6 \beta+1}} C_{2}=0
$$

Solving these two algebraic equations by the elimination method, for $C_{1}$ and $C_{2}$, we get that $C_{1}=C_{2}=0$.

Therefore, Equation (3.5) has the unique solution (for the uniqueness of the solution, see Zwillinger, 1992, p. 51):

$$
\phi(t)=-\frac{(1-\ell t)}{(1-\beta)\left(1-\rho_{1} t\right)}, \quad t \in(0 ; 1), \beta \in(0 ; 1), \text { and } \rho_{1} \in[0,1) .
$$

Consequently, we have uniquely $\left(\phi(s)=-1 / P_{X_{1}}(s)\right)$

$$
P_{X_{1}}(s)=\frac{(1-\beta)\left(1-\rho_{1} s\right)}{(1-\ell s)}, \quad|s| \leq 1, \beta \in(0 ; 1), \text { and } \rho_{1} \in[0,1) .
$$

Therefore, $X_{1} \sim M B G\left(\beta, \rho_{1}\right)$. This completes the proof of Theorem 3.2.

Remark 3.2 If $\rho_{1}=0$; Theorem 3.2 reduces to Theorem 4 of Ghitany and Gharib (2005) concerning the case of geometric distribution.

The third characterization of the Markov-Bernoulli geometric distribution related to the random sum (1.3) is expressed in terms of the first four moments of $Z$ and the first two moments of $X_{1}$.

Theorem 3.3 Let $Y_{1} \sim M B G(\alpha, \rho)$; for some $\alpha \in(0 ; 1)$ and $\rho \in[0,1]$, and let $X_{1}$ be an arbitrary non-negative integer-valued $r v$ with $p_{0}=P\left(X_{1}=0\right)<1$. Then $X_{1} \sim M B G\left(\beta, \rho_{1}\right)$, for some $\beta \in(0 ; 1)$, and $\rho_{1} \in[0,1]$, if and only if

$$
\begin{gathered}
3\left(\mu_{4}-2 \mu_{3}-\mu_{2}+2 \mu_{1}\right)\left(\mu_{2}-\mu_{1}\right)-4\left(\mu_{3}-3 \mu_{2}+2 \mu_{1}\right)\left(\mu_{3}-\mu_{1}\right)=0, \\
\mu_{3}-3 \mu_{2}+2 \mu_{1}-3\left(\mu_{2}-\mu_{1}\right) v_{1}=\frac{6 \beta D \rho_{1}(t-D)}{(1-\ell)\left(1-\rho_{1} t\right)(1-D)^{2}},
\end{gathered}
$$

and

$$
v_{2}-v_{1}\left(2 v_{1}+1\right)=\frac{2 \beta \rho_{1}}{(1-\ell)\left(1-\rho_{1} t\right)},
$$

where $\mu_{i}=E\left(Z^{i}\right), i=1,2,3,4$, with $\mu_{4}<\infty, v_{j}=E\left(X_{1}^{j}\right), j=1 ; 2, D=\rho_{1} t+\left(1-\rho_{1} t\right) \beta, t=\rho+(1-\rho) \alpha$ and $\ell=\rho_{1}+\left(1-\rho_{1}\right) \beta$.

Proof. Necessity: Suppose $X_{1} \sim \mathrm{MBG}\left(\beta, \rho_{1}\right)$ for some $\beta \in(0 ; 1)$, and $\rho_{1} \in[0,1]$. Then according to Lemma $1.2, Z$ $\sim \mathrm{MBG}\left(\beta, \rho_{2}\right)$, for some $\beta \in(0 ; 1)$ and $\rho_{2}=\rho_{1} t, t=\rho+(1-\rho) \alpha$. Now, it is straight forward to see that each of conditions (3.6)-(3.8) is satisfied upon substituting

$$
\begin{gathered}
\mu_{1}=\frac{\beta}{1-D}, \quad \mu_{2}=\frac{\mu_{1}(1+D)}{1-D}, \quad \mu_{3}=\frac{\mu_{1}\left(D^{2}+4 D+1\right)}{(1-D)^{2}}, \quad \mu_{4}=\frac{\mu_{1}\left(D^{3}+11 D^{2}+11 D+1\right)}{(1-D)^{3}}, \\
v_{1}=\frac{\beta}{1-\ell}, \quad v_{2}=\frac{v_{1}(1+\ell)}{1-\ell},
\end{gathered}
$$

where, $D=\rho_{1} t+\left(1-\rho_{1} t\right) \beta, t=\rho+(1-\rho) \alpha$ and $\ell=\rho_{1}+\left(1-\rho_{1}\right) \beta$. 
Sufficiency: Suppose conditions (3.6)-(3.8) are satisfied. These and (iii) of Lemma 2.1, imply that

$$
t^{3} \phi^{\prime \prime \prime}(t)+3 \mu_{1} t^{2} \phi^{\prime \prime}(t)+3\left(\mu_{2}-\mu_{1}\right) t \phi^{\prime}(t)+\left(\mu_{3}-3 \mu_{2}+2 \mu_{1}\right) \phi(t)=-\frac{1}{4}\left(\mu_{4}-2 \mu_{3}-\mu_{2}+2 \mu_{1}\right)(1-t), \quad t \in(0 ; 1)
$$

subject to the initial conditions $\phi(1)=-1, \phi^{\prime}(1)=v_{1}=\frac{\beta}{1-\ell}$,

$$
\phi^{\prime \prime}(1)=v_{2}-v_{1}\left(2 v_{1}+1\right)=\frac{2 \beta \rho_{1}}{(1-\ell)\left(1-\rho_{1}\right)},
$$

where $\ell=\rho_{1}+\left(1-\rho_{1}\right) \beta, \rho_{1} \in[0,1), \mu_{3}-3 \mu_{2}+2 \mu_{1}=E[Z(Z-1)(Z-2)]>0, \mu_{2}-\mu_{1}>0$ and $\mu_{1}>0$.

From conditions (3.3), (3.6) and (3.7), we obtain:

$$
\mu_{3}-\mu_{1}=\frac{3\left(\mu_{2}-\mu_{1}\right)\left(\mu_{2}+\mu_{1}\right)}{2 \mu_{1}}, \quad \mu_{3}-3 \mu_{2}+2 \mu_{1}=\frac{6 \beta D^{2}}{(1-D)^{3}}, \quad \mu_{4}-2 \mu_{3}-\mu_{2}+2 \mu_{1}=\frac{24 \beta D^{2}}{(1-D)^{4}} .
$$

Therefore, (3.9) can be rewritten as:

$$
t^{3} \phi^{\prime \prime \prime}(t)+\frac{3 \beta t^{2}}{(1-D)} \phi^{\prime \prime}(t)+\frac{6 \beta D t}{(1-D)^{2}} \phi^{\prime}(t)+\frac{6 \beta D^{2}}{(1-D)^{3}} \phi(t)=-\frac{6 \beta D^{2}}{(1-D)^{4}}(1-t), \quad t \in(0 ; 1)
$$

with initial conditions $\phi(1)=-1, \phi^{\prime}(1)=v_{1}=\frac{\beta}{1-\ell}$,

$$
\phi^{\prime \prime}(1)=v_{2}-v_{1}\left(2 v_{1}+1\right)=\frac{2 \beta \rho_{1}}{(1-\ell)\left(1-\rho_{1}\right)} .
$$

Finally, Equation (3.10) with the above initial conditions has the unique solution (for the uniqueness of the solution, see Zwillinger, 1992, p. 51):

$$
\phi(t)=-\frac{(1-\ell t)}{(1-\beta)\left(1-\rho_{1} t\right)}, \quad t \in(0 ; 1), \beta \in(0 ; 1), \text { and } \rho_{1} \in[0,1) .
$$

Consequently, we have uniquely $\left(\phi(s)=-1 / P_{X_{1}}(s)\right)$

$$
P_{X_{1}}(s)=\frac{(1-\beta)\left(1-\rho_{1} s\right)}{(1-\ell s)}, \quad|s| \leq 1, \beta \in(0 ; 1), \text { and } \rho_{1} \in[0,1) .
$$

Therefore, $X_{1} \sim M B G\left(\beta, \rho_{1}\right)$. This completes the proof of Theorem 3.3.

Remark 3.3 If $\rho_{1}=0$; Theorem 3.3 reduces to Theorem 5 of Ghitany and Gharib (2005) concerning the case of geometric distribution.

\section{Concluding Remarks}

1) From the "if" part of Theorems 3.1-3.3 and according to Theorem 1.1, It follows that when $X_{1}$ has the MBG distribution then the random sum $Z$ and the summands have distributions of the same type and in this case the summands are called N-sum stable (Satheesh et al., 2002). This result is valid, also, as a consequence of the fact that geometric random sums are stable in the same sense.

2) Each of the three nonhomogeneous differential equations used in the characterizations of the Markov-Bernoulli geometric distribution given in this paper has a linear nonhomogeneous term. The solution associated with each of these differential equations under certain initial condition(s) is essentially its particular solution.

3) We may note that the condition of Theorem 3.1 involves only the moments of $Z$ while the conditions of Theorem 3.2 and Theorem 3.3 involve both the moments of $Z$ and $X_{1}$ : Indeed, this is due to the nature of the initial conditions used in the sufficiency part of the proofs of these theorems.

\section{Acknowledgements}

The authors wish to express their gratitude to the editor and anonymous referees for their helpful comments which led to a significantly improved presentation. 


\section{References}

Anis, A. A., \& Gharib, M. (1982). On the Bernoulli Markov Sequences. 17th Ann. Conf. Statist. Math. Inst. Statist. Studies Res., 2, 1-21. Egypt.

Arvidsson, H., \& Francke, S. (2007). Dependence in Non-Life Insurance. U.U.D.M. Project Report: 23.

C̆ekanavičius, V., \& Vellaisamy, P. (2010). Compound Poisson and Signed Compound Approximations to the Markov Binomial Law. Bernoulli, 16(4), 1114-1136. http://dx.doi.org/10.3150/09-BEJ246

Chang, Y., \& Zeiterman, D. (2002). Sums of Dependent Bernoulli Random Variables and Disease Clustering. Stat. Prob. Letters, 57, 363-373.

Dimitrov, B. , Khalil, Z. , Kolev, N., \& Petrov, P. (1991). On the Optimal Total Processing Time Using Checkpoints. IEEE Trans. Software Eng., 17(5), 436-442. http://dx.doi.org/10.1109/32.90446

Duda, A. (1983). The Effects of Checkpointing on Program Execution Time. Inform Process Letters, 16(5), 221-229. http://dx.doi.org/10.1016/0020-0190(83)90093-5

Edwards, A. W. F. (1960). The Meaning of Binomial Distribution. Nature, $186,1074$. http://dx.doi.org/10.1038/1861074a0

Gharib, M., Nassar, M. M., \& Ramadan, M. M. (2003). On Random Sum Characterization. Proc. Math. Phys. Soc. Egypt, 79, 349-353.

Gharib, M., Ramadan, M. M., \& Al-Ajmi, Kh. A. H. (2014). Characterization of Makov-Bernoulli Geometric Distribution Related to Random Sums. J. Math. \& Stat., $10(2), \quad 186-191$. http://dx.doi.org/10.3844/jmssp.2014.186.191

Gharib, M., \& Yehia, A. Y. (1987). A Limit Theorem for the Sum of n-Markov-Bernoulli Random Variables. Egypt. Stat. J., 31(1), 53-61.

Ghitany, M. E., \& Gharib, M. (2005). New Characterizations of the Geometric Distribution Related to Random Sums. Int. J. Appl. Math., 18(2), 223-234.

Gnedenko, B. V., \& Korolev, V. Yu. (1996). Random Summation: Limit Theorems and Applications. New York: CRC.

Inal, C., (1987). On the Limiting Distribution for Bernoulli Trials Within a Markov-Chain Context. Comm. Fac. Sci. Univ. Ankara, Ser. A, 36(2), 123-129.

Khalil, Z., Dimitrov B., \& Dion, J. P. (1991). A Characterization of the Geometric Distribution Related to Random Sums. Comm. Stat.- Stochastic Models, 7(2), 321-326. http://dx.doi.org/10.1080/15326349108807192

Koren, J., Koren, Z., \& Su, S. (1986). Analysis of a Class of Recovery Procedures. IEEE Trans. Comput. C-35, 8, 703-712.

Maillart, L. M., Richard Cassady, C., \& Honeycutt, J. (2008). A Binomial Approximation of Lot Yield under Markov Modulated Bernoulli Item Yield. IIE Trans., 40(4), 459-467. http://dx.doi.org/10.1080/07408170701592507

McCall, J. J. (1971). A Markovian Model of Income Dynamics. JASA, 66(335), 439-447.

Milne, R. K., \& Yeo, G. F. (1989). Random Sum Characterizations. Math Sci., 14(2), 120-126.

Minkova, L. D., \& Omey, E. A. (2011). A New Markov-Binomial Distribution. HUB Research papers. Economics \& Management, 24, 1-14.

Omey, E., Santos, J., \& Van Gulck, S. (2008). A Markov-Binomial Distribution. Applicable Analysis \& Discrete Math., 2(1), 38-50. http://dx.doi.org/10.2298/AADM0801038O

Özekici, S. (1997). Markov Modulated Bernoulli Process. Math. Meth. Oper. Res., 45, 311-324.

Özekici, S., \& Soyer, R. (2003). Bayesian Analysis of Markov Modulated Bernoulli Processes. Math. Meth. Oper. Res., 57, 125-140. http://dx.doi.org/10.1007/s001860200268

Pacheco, A., Tang, L. Ch., \& Prabhu, N. U. (2009). Markov-Modulated Processes and Semiregenerative Phenomena (pp. 224). World Scientific Publ. Co. Pte. Ltd. UK. 
Pedler, P. J. (1980). Effects of Dependence on the Occupation Time in a Two-State Stationary Markov Chain. J. Amer. Statist. Assoc., 75(371), 739-764. http://dx.doi.org/10.1080/01621459.1980.10477545

Pires, R. M., \& Diniz, C. A. R. (2012). Correlated Binomial Regression Models. Comp. Stat. \& Data analysis, 56(8), 2513-2525. http://dx.doi.org/10.1016/j.csda.2012.02.004

Satheesh, S., Nair, N. H., \& Sandhya, E. (2002). Stability of Random Sums. Stoch. Modell. Appl., 5(1), 17-26.

Switzer, P. (1967). Reconstructing Patterns from Sample Data. Ann. Math. Statist., 38(1), $138-154$. http://dx.doi.org/10.1214/aoms/1177699064

Switzer, P. (1971). Mapping a Geographically Correlated Environment. Proc. 1969, Int. Symp. Statist. Ecology, 1, 235-270. Yale Univ.

Wang, Y. H. (1981). On the Limit of the Markov Binomial Distribution. J. Appl. Prob., 18, 937-942.

Xekalaki, E., \& Panaretos, J. (2004). A Binomial Distribution with Dependent trials and Its Use in Stochastic Model Evaluation. Comm. Statist. Theo. Meth., 33(12), 3043-3058.

Yehia, A. Y., \& Gharib, M. (1993). Characterizations of the Markov-Bernoulli Geometric Distribution. Microelectronics \& Reliab., 33(4), 497-499. http://dx.doi.org/10.1016/0026-2714(93)90317-R

Zwillinger, D. (1992). Handbook of Differential Equations (2nd ed.). New York: Academic Press.

\section{Copyrights}

Copyright for this article is retained by the author(s), with first publication rights granted to the journal.

This is an open-access article distributed under the terms and conditions of the Creative Commons Attribution license (http://creativecommons.org/licenses/by/3.0/). 